\title{
Indicadores sociodemográficos, sexualidad y embarazo en adolescentes de Santa Marta-Colombia
}

\author{
Sara Josefina Zabarain-Cogollo y Martha P. Fernández-Daza \\ Universidad Cooperativa de Colombia (Colombia)
}

\begin{abstract}
La presente investigación descriptiva transaccional, no experimental, pretende determinar los indicadores sociodemográficos, sexualidad y embarazo en 88 madres pertenecientes a programas de prevención del Instituto Colombiano de Bienestar Familiar (ICBF) Centro Zonal Norte. Para la recolección de datos se utilizó una ficha sociodemográfica elaborada por los investigadores, teniendo en cuenta lineamientos de otros trabajos. El análisis se realizó a través del software estadístico SPSS versión 25 y se utilizó el estadígrafo Chi-Cuadrado para la obtención de la frecuencia y el porcentaje. Se siguieron todas las normativas establecidas por los criterios de la resolución 8430/93, expedida por el Ministerio de Salud de Colombia, y la Ley 1090 del Psicólogo. Los resultados muestran que los indicadores sociodemográficos y de sexualidad asociados al embarazo adolescente son: gestación a edad temprana, baja escolaridad, estrato socioeconómico bajo, no utilización de los métodos anticonceptivos, embarazo no planificado.
\end{abstract}

Palabras claves: Embarazo, adolescencia, sexualidad.

Sociodemographic indicators, sexuality and pregnancy in adolescents in Santa MartaColombia. This non-experimental transactional descriptive research aims to determine the sociodemographic, sexuality and pregnancy indicators in 88 mothers belonging to prevention programs of the Colombian Family Welfare Institute (ICBF) Centro Zonal Norte. For the data collection, a sociodemographic record prepared by the researchers was used, taking into account guidelines from other works. The analysis was carried out through the statistical software SPSS version 25 and the Chi-Square statistic was used to obtain the frequency and percentage. All the regulations established by the criteria of resolution $8430 / 93$, issued by the Ministry of Health of Colombia, and Law 1090 of the Psychologist were followed. The results show that sociodemographic and sexuality indicators associated with teenage pregnancy are: early pregnancy, low schooling, low socioeconomic status, non-use of contraceptive methods, unplanned pregnancy.

Keywords: Pregnancy, adolescence, sexuality.

\footnotetext{
Correspondencia: Sara Josefina Zabarain Cogollo. Universidad Cooperativa de Colombia, Sector Troncal del Caribe. Vía Mamatoco. C.P.: 470003. Santa María (Colombia). E-mail: Sara.Zabarain@campusucc.edu.co
} 
Estado actual de los indicadores sociodemográficos del embarazo en Latinoamérica y el Caribe

La Organización Mundial de la Salud (OMS) define la adolescencia como un periodo de crecimiento y desarrollo humano entre los 10 y 19 años de edad. En Colombia la Ley 1098 de noviembre del 2006, considera adolescentes a las personas con edades comprendidas entre los 12 y 18 años.

El embarazo en la adolescencia es una problemática que afecta la población mundial. Según la división de población de las Naciones Unidas (2019), la maternidad entre las adolescentes de 10 a 14 años de edad es un reto para la comunidad internacional y es uno de los objetivos del desarrollo sostenible, no obstante, este periodo es pasado por alto por investigadores y políticos.

La situación demográfica de las madres adolescentes en Iberoamérica se encuentra en torno a un contexto de pobreza, falta de educación, soltería e inestabilidad emocional. Esta situación implica que las adolescentes queden desprovistas de lo básico y necesario para la sobrevivencia.

Este contexto no es ajeno a los países de Latinoamérica, ya que existe dificultad en la socialización de la información pertinente a todos los grupos poblacionales afectados (madre adolescente, familia, pareja) que permita realizar una prevención de factores de riesgo afectivos y del desarrollo.

La OMS (2018) indica que unos 16 millones de muchachas de 15 a 19 años y aproximadamente un millón de niñas menores de 15 años tienen hijos, la mayoría en países de ingresos bajos y medianos. De igual manera la OPS, la OMS, UNFPA y la Unicef (2018), consideran que el embarazo en adolescentes afecta la trayectoria de la vida de las adolescentes, interfiere en su desarrollo psicosocial, vulnera la salud de las jóvenes y sus hijos, y tiene implicaciones para su educación y posterior oportunidad de empleo.

La UNFPA (2018) menciona que el Fondo de población de las Naciones Unidas indica que lo adecuado sería que los embarazos fuesen planificados, más sin embargo, esta realidad no es aplicable a América Latina y el Caribe ya que se ha incrementado los embarazos en niñas menores de 15 años, lo que pareciera ser propio de estas regiones. Según la UNFPA (2018) del 2010 al 2015, se observa una tasa de fecundidad de 66.6 nacimientos en adolescentes de América Latina y el Caribe.

En Brasil se observan 67.0 nacimientos, en Argentina 64.0 nacimientos, en Chile 50.4 nacimientos, en Colombia 57.7 nacimientos, en Costa Rica 59.1 nacimientos, en Cuba 50.6 nacimientos, en República Dominicana 100.6 nacimientos, en Guatemala 78.6 nacimientos, en Jamaica 60.8 nacimientos, en México 66.0 nacimientos, en Perú 52.1 nacimientos y en Uruguay 58.0 nacimientos.

El Observatorio de la Juventud (2017), señala que los embarazos en adolescentes en República Dominicana corresponden al 22\% de los nacimientos y son un 
tercio más alto que el promedio de los países de América Latina y el Caribe. La UNFPA, América Latina y el Caribe (2019) indican que en el año 2017 en el Salvador se registraron 19.190 embarazos en niñas y adolescentes entre los 10 y 19 años; 53 niñas o adolescentes embarazadas por día. La mesa de concertación para la lucha contra la pobreza (2018), determinaron que, en el Perú, entre enero y septiembre del 2017, atendieron 1. 645 partos en menores de 15 años de edad.

Los factores sociodemográficos comunes en todos estos países son: estrato socioeconómico bajo y pobreza, baja escolaridad, la pareja de la madre adolescente es mayor de edad (20 años o más), la cultura y mitos sobre la sexualidad, conocimiento de métodos anticonceptivos, pero acceso limitado a estos, poca comunicación familiar, la tipología familiar, inestabilidad conyugal (Blanquet-García, Montoya-Cázares, y Carranza-Lira, 2016; García y González, 2018; Huanco, Ticona, Ticona, y Huanco, 2012). Estos aspectos sociodemográficos son similares para la población de madres adolescentes en Colombia (Mazuera-Arias, Trejos-Herrera, y Reyes-Ruiz, 2017; Carvajal, Valencia, y Rodríguez, 2017; Gómez-Mercado y Mejía-Sandoval, 2017; Rojas, Méndez, y Montero, 2016; Pinzón-Rondon, Ruiz-Sternberg, Aguilera-Otalvaro, y Abril-Basto, 2018; Tafur-Bonilla, Barbosa-Campos, y Méndez-Ortiz, 2018).

\section{Sexualidad en la adolescencia}

En la adolescencia el cuerpo de niño es dejado atrás, se presenta la maduración genital y la capacidad reproductora y el despertar de lo orgásmico-genital (Urribarri, 2016). Se presenta un despertar de la sexualidad en la que muchos jóvenes experimentan y descubren la genitalidad sin tener una orientación adecuada.

Las relaciones sexuales precoces en la adolescencia colocan en riesgo la integridad del cuerpo de la joven al exponerse a un embarazo o problemas de salud, pero también producen cambios psíquicos y emocionales al experimentar sentimientos de culpa. En ocasiones la decisión de tener relaciones sexuales tempranas de parte de las adolescentes se encuentra asociado al deseo de complacer a la pareja, pero también se encuentra ligado a la curiosidad y al deseo inconsciente de independencia al tomar decisiones con respecto a su cuerpo (Blos, 2011; Mendoza, Claros, y Peñaranda, 2016; López, 2014).

\section{Embarazo en la adolescencia}

El embarazo en la adolescencia implica un cambio en el proyecto de vida y las vivencias que son típicas de este momento evolutivo. Las mayores dificultades relacionadas con la aceptación del embarazo de la madre adolescente se relacionan con el rechazo de parte de sus padres debido a la gestación, la falta de apoyo de parte de su pareja o complicaciones de salud. 
Una vez ha pasado este primer momento, la madre adolescente parece vincularse emocionalmente con su hijo sin dificultad. En algunos casos cuando existen conflictos en el interior de la familia la madre adolescente se aferra a esta nueva vida con amor y deseo de protección incondicional; "El hijo es el nuevo "motivo para" del curso de vida” (Pacheco-Sánchez, 2015, p. 58).

Una vez nace él bebe se generan cambios en torno a todo lo que corresponden los hábitos y costumbres, y es cuando la joven madre requiere todo el apoyo de su entorno familiar. Si la red de apoyo le falla, existen varias posibilidades: el amor hacia su hijo se intensifica, rechaza a su hijo o delega el total cuidado al padre, a sus padres o a terceros. La postura del padre adolescente frente al embarazo de su pareja parece asociarse a "los ideales relacionados con ser un mejor padre de lo que fueron sus propios padres con ellos” (Botero-Botero y Castrillón-Osorio, 2015, p. 92).

Por todo lo anteriormente mencionado este proyecto busco la caracterización de los indicadores sociodemográficos, la sexualidad y el embarazo en adolescentes, encaminado en dos fases: una primera fase cuyo propósito fue establecer un diagnóstico que permitiera identificar factores de riesgo y protección de la sexualidad, el embarazo y la paternidad en adolescencia, y se tiene planificada una segunda fase correspondiente a la elaboración de una propuesta psicoeducativa de prevención en donde se incluye a las adolescentes, su pareja y sus familias.

Se encuentra enmarcado en lo que contempla el numeral 8 del artículo 46 de la Ley Colombiana 1098. De igual forma al intervenir en este problema, se realiza la prevención de riesgos afectivos en la población de primera infancia como son: la deprivación afectiva, los trastornos reactivos del vínculo, problemas de trastornos alimentarios, entre otros.

Se contó con el apoyo del centro Zonal Norte del ICBF (Instituto Colombiano de Bienestar Familiar) de la ciudad de Santa Marta y los resultados serán socializados a las instituciones del Sistema Nacional de Bienestar Familiar que confluyen en la Mesa de infancia, adolescencia y fortalecimiento Familiar del distrito de Santa MartaColombia y en la Submesa de prevención de Embarazo en Adolescentes, con el objeto de sensibilizar e incentivar un accionar armónico para las intervenciones de esta problemática.

\section{MÉTODO}

\section{Participantes}

La muestra estuvo formada por 88 madres adolescentes que pertenecen a programas de prevención del ICBF, con edades entre los 13 y 18 años, con hijos natos y no natos. 


\section{Procedimiento}

Se utilizó una ficha sociodemográfica que permitiera la caracterización de datos generales y específicos sobre la sexualidad y embarazo, diseñada por los investigadores.

El proyecto tuvo el siguiente proceso:

1) Visita a las instituciones que hacen parte de la Submesa distrital de prevención de embarazo en adolescentes.

2) Socialización de la propuesta con las madres adolescentes y, la firma del consentimiento informado.

3) Revisión de documentos para el diseño de la ficha sociodemográfica.

4) Aplicación del instrumento.

5) Análisis de resultados.

6) Elaboración de productos y divulgación de resultados.

\section{Análisis de datos}

El tipo de investigación a utilizada fue descriptivo con un diseño transversal no experimental. Los datos se recolectaron en un tiempo único (octubre del 2017 a noviembre del 2018).

El análisis de datos se realizó con el software SPSS 25, utilizando el estadígrafo Chi-cuadrado para el análisis descriptivo y en porcentajes de los resultados del instrumento.

\section{RESULTADOS}

\section{Indicadores Sociodemográficos}

Se observa que la mayoría de las adolescentes tenía 18 años. En cuanto al estado civil, se puede ver que 59 de ellas convive en unión libre.

Sobre el estrato socioeconómico de las madres adolescentes la mayoría pertenecía al estrato 1. En cuanto a la escolaridad, 47 de ellas no completó la secundaria. Por otro lado, se observa que 34 adolescentes, tenían familia nuclear, 29 monoparental y el resto familia reconstituida (Tabla 1). 
Tabla 1. Indicadores Sociodemográficos

\begin{tabular}{|c|c|c|c|}
\hline & & Frecuencia & Porcentaje \\
\hline \multirow{6}{*}{ Edad } & 13 & 1 & 1.1 \\
\hline & 14 & 2 & 2.3 \\
\hline & 15 & 8 & 9.1 \\
\hline & 16 & 18 & 20.5 \\
\hline & 17 & 26 & 29.5 \\
\hline & 18 & 33 & 37.5 \\
\hline \multirow{3}{*}{ Estado civil } & Casada & 2 & 2.3 \\
\hline & Soltera & 27 & 30.7 \\
\hline & Unión Libre & 59 & 67 \\
\hline \multirow{3}{*}{ Estrato Socioeconómico } & 1 & 77 & 87.5 \\
\hline & 2 & 7 & 8 \\
\hline & 3 & 3 & 3.4 \\
\hline \multirow{6}{*}{ Escolaridad } & No lo conoce & 1 & 1.1 \\
\hline & Primaria completa & 4 & 4.5 \\
\hline & Primaria incompleta & 5 & 5.7 \\
\hline & Secundaria completa & 30 & 34.1 \\
\hline & Secundaria incompleta & 47 & 53.4 \\
\hline & Técnico & 1 & 1.1 \\
\hline \multirow{4}{*}{ Tipo de familia } & Otros & 1 & 1.1 \\
\hline & Nuclear & 34 & 38.6 \\
\hline & Monoparental & 29 & 33 \\
\hline & Reconstituida & 24 & 0 \\
\hline
\end{tabular}

Fuente: Elaboración propia.

\section{Sexualidad}

Sobre la sexualidad se halló que 29 de las adolescentes tuvieron su primera relación sexual cuando tenían 15 años; Al preguntarles si las relaciones sexuales fueron con su consentimiento 84 adolescentes respondieron afirmativamente.

Tabla 2. Sexualidad

\begin{tabular}{|c|c|c|c|}
\hline & & Frecuencia & Porcentaje \\
\hline \multirow{8}{*}{$\begin{array}{l}\text { Edad de Inicio de } \\
\text { Relaciones Sexuales }\end{array}$} & 11 & 2 & 2.3 \\
\hline & 12 & 1 & 1.1 \\
\hline & 13 & 8 & 9.1 \\
\hline & 14 & 25 & 28.4 \\
\hline & 15 & 29 & 33 \\
\hline & 16 & 12 & 13.6 \\
\hline & 17 & 10 & 11.4 \\
\hline & 18 & 1 & 1.1 \\
\hline \multirow{2}{*}{$\begin{array}{l}\text { Consentimiento de } \\
\text { Relaciones Sexuales }\end{array}$} & si & 84 & 95.5 \\
\hline & no & 4 & 4.5 \\
\hline \multirow{2}{*}{$\begin{array}{l}\text { Conoce Métodos de } \\
\text { Planificación Familiar }\end{array}$} & si & 78 & 88.6 \\
\hline & no & 10 & 11.4 \\
\hline \multirow{2}{*}{$\begin{array}{l}\text { Utiliza Método de } \\
\text { planificación familiar }\end{array}$} & si & 52 & 59.1 \\
\hline & no & 36 & 40.9 \\
\hline \multirow{6}{*}{$\begin{array}{l}\text { Método Planificación } \\
\text { Familiar que utiliza }\end{array}$} & Coito interrumpido & 19 & 21.6 \\
\hline & Barrera & 2 & 2.3 \\
\hline & Hormonales & 52 & 59.1 \\
\hline & Ninguno & 10 & 11.4 \\
\hline & No hormonales permanentes & 2 & 2.3 \\
\hline & No hormonales reversibles & 3 & 3.4 \\
\hline
\end{tabular}

Fuente: Elaboración propia. 
En cuanto a la frecuencia con que las adolescentes utilizan los métodos de planificación familiar, se aprecia que más de la mitad de las adolescentes (52) respondieron que si los utilizan. Así mismo, el método de planificación familiar que más utilizan las adolescentes del estudio son los hormonales, seguidos de coito interrumpido y 10 no utilizan ninguno (Tabla 2).

\section{Embarazo}

En lo que respecta a la edad que tenían durante su primer embarazo, la mayoría de las adolescentes afirmó que a los 15 años. También se observa que 51 adolescentes indicaron que no fue un embarazo deseado, y 66 de ellas señalaron que este es su primer embarazo. Sobre el número de embarazos 67 de ellas, solo ha tenido un embarazo y 68 de ellas consideran que no fue un embarazo planificado. Y, por último, las adolescentes percibieron apoyo de su compañero durante el embarazo (Tabla 3).

Tabla 3. Embarazo

\begin{tabular}{|c|c|c|c|}
\hline & & Frecuencia & Porcentaje \\
\hline \multirow{7}{*}{ Edad de primer embarazo } & 12 & 1 & 1.1 \\
\hline & 13 & 4 & 4.5 \\
\hline & 14 & 12 & 13.6 \\
\hline & 15 & 26 & 29.5 \\
\hline & 16 & 23 & 26.1 \\
\hline & 17 & 16 & 18.2 \\
\hline & 18 & 6 & 6.8 \\
\hline \multirow{2}{*}{ Fue o es un embarazo deseado } & si & 37 & 40.9 \\
\hline & no & 51 & 58 \\
\hline \multirow{2}{*}{ Es este su primer embarazo } & si & 66 & 75 \\
\hline & no & 22 & 25 \\
\hline \multirow{3}{*}{ Número de embarazos } & 1 & 67 & 76.1 \\
\hline & 2 & 18 & 20.5 \\
\hline & 3 & 3 & 3.4 \\
\hline \multirow{3}{*}{ Fue un embarazo planificado } & si & 18 & 20.5 \\
\hline & no & 68 & 77.3 \\
\hline & Perdidos & 2 & 2.3 \\
\hline \multirow{3}{*}{$\begin{array}{l}\text { Ha recibido apoyo afectivo de parte } \\
\text { de su compañero durante el embarazo }\end{array}$} & si & 68 & 77.3 \\
\hline & no & 19 & 21.6 \\
\hline & Perdidos & 1 & 1.1 \\
\hline
\end{tabular}

Fuente: Elaboración propia.

Al preguntarles si ha recibido apoyo afectivo de parte de su compañero durante el embarazo el $77.3 \%$ de las madres adolescentes indicaron que sí (Tabla 4).

Tabla 4. Ha recibido apoyo afectivo de parte de su compañero durante el embarazo

\begin{tabular}{|c|c|c|c|}
\hline & & Frecuencia & Porcentaje \\
\hline & $\mathrm{Si}$ & 68 & 77.3 \\
\hline & No & 19 & 21.6 \\
\hline & Total & 87 & 98.9 \\
\hline Perdidos & Sistema & 1 & 1.1 \\
\hline Total & & 88 & 100.0 \\
\hline
\end{tabular}




\section{DISCUSIÓN Y CONCLUSIONES}

A continuación, se presenta el análisis, discusión y conclusiones de los resultados de este estudio con la población de madres adolescentes.

El $87 \%$ de las adolescentes estaban entre los 16 y 18 años. Estudios recientes exponen que alrededor del $15 \%$ de los embarazos en América Latina y el Caribe corresponden a menores de 20 años (OPS y OMS, 2018).

En relación con su estado civil más de la mitad de las madres adolescentes se encontraba en unión libre (57) y 27 eran solteras.

Las madres adolescentes pertenecen a los estratos más bajos en Colombia (1 y 2) y la mayor proporción era del estrato 1 (77 madres adolescentes). En este sentido, los resultados concuerdan con la UNFPA (2018) que plantean que las adolescentes generalmente viven en los estratos más pobres.

En lo que respecta a la escolaridad 47 adolescentes no culminaron la secundaria y 30 si lo habían hecho, ninguna había iniciado estudios universitarios. La tasa de deserción escolar de las adolescentes muestra que pudieron deberse a la responsabilidad temprana de un embarazo. En este sentido los resultados son coincidentes con la Encuesta Nacional de Demografía y Salud de Colombia (2015) y con aquellos que expresan que las madres adolescentes tienen menor nivel educativo (UNFPA, 2018).

El tipo de familia del $38.6 \%$ era nuclear, mientras que el $33 \%$ tenía familia monoparental y el resto familia reconstituida.

Sobre la sexualidad 29 adolescentes tuvieron su primera relación sexual cuando tenían 15 años; 25 cuando tenían 14 años; 12 a los 16 años y 10 a los 17 años. Es decir, el 95\% de la muestra del estudio se inició sexualmente entre los 13 y 18 años. Los presentes hallazgos pudieran explicarse con evidencias departamentales donde explícitamente se incluye al Magdalena como uno de los departamentos donde las mujeres y los hombres afirman haber recibido menos información sobre temas de sexualidad (mujeres $75.7 \%$ y hombres $72.7 \%$ ). asimismo, el departamento lidera las mayores cifras donde los entrevistados señalan que no han participado en actividades de educación sexual en los plateles educativos (Encuesta Nacional de Demografía y Salud de Colombia, 2015). Cabe reflexionar que el colegio no tiene un papel activo en la educación sexual de las adolescentes; papel que debe ejercer debido a que en Colombia se estableció que los colegios debían impartir cátedras de educación sexual; también se asemeja a estudios que señalan que la edad del primer coito de adolescentes de América Latina y el Caribe es alrededor de los 15 a 16 años, pero también hay casos entre los 10 y 12 años (Mendoza et al., 2016; Sanabria y Fernández, 2019). 
El 95,5\% de las adolescentes tuvieron relaciones sexuales con su consentimiento. Contrario a lo planteado por Castro y López (2014) quienes mencionan que cuando las adolescentes son mayores de 15 años puede suceder que tengan relaciones sexuales no consentidas, obligadas por sus mismas parejas. Sobre los métodos de planificación familiar 78 adolescentes (59.1\%) afirmaron que los conocen. Datos discordantes con los resultados de la Encuesta Nacional de Demografía y Salud de Colombia (2015) que afirman que las adolescentes que tienen entre 13 y 19 años el conocimiento de algún método anticonceptivo está por encima del 97 por ciento. No obstante, es preciso recordar que el presente estudio solo incluyó adolescentes hasta los 18 años y que el tamaño muestral es menor. Mientras que en la encuesta nacional participaron 38.718 mujeres entre 13 y 49 años y 35.783 hombres entre los 13 a 59 años.

En esta línea los hallazgos relacionados con los métodos de planificación familiar ante los cuales el 59, $1 \%$ de las madres adolescentes indicaron que utilizan el método hormonal y el $21.6 \%$ el coito interrumpido. No obstante, el $40.9 \%$ no utilizan ningún método. Al respecto, la Encuesta Nacional de Demografía y Salud de Colombia (2015) señala que el $69.9 \%$ de las madres adolescentes cuyas edades estaban entre 15 y 19 años no usaban métodos anticonceptivos. La mayoría de las adolescentes manifiesta que fue su madre la que le indico acerca de los métodos de planificación familiar, pero existe la posibilidad de que la información suministrada haya sido general e informal, y no se les haya dado con la rigurosidad y claridad que se requiere. Cabe resaltar que existe un tema cultural relacionado con decir a los hijos que deben "cuidarse", pero no se les capacita realmente en el tema. En lo que respecta al embarazo 26 adolescentes señalaron que tuvieron su primer embarazo a los 15 años, por lo cual, el $87.4 \%$ de la muestra tuvo su primer embarazo entre los 14 y 17 años. Según la OMS, la OPS, la UNFPA y la UNICEF en 2016 en Colombia hay estudios que señalan que entre 2005 y 2015 la proporción de adolescentes entre los 15 y 19 años que se encuentran embarazadas o son madres se redujo del $20.5 \%$ al $17.4 \%$. Si tenemos en cuenta solo las menores de 15 años, el $19.2 \%$ de la muestra de madres tuvieron su primer embarazo entre los 12 y 14 años. Resultados concordantes con datos de la OMS, la OPS, la UNFPA y la UNICEF en 2016 que reportan que $2 \%$ de las mujeres en edad fecunda en América Latina y el Caribe tuvieron su primer parto antes de los 15 años. Llama la atención que más de la mitad de la muestra de madres adolescentes (51) indicaron que no fue un embarazo deseado y el 75\% expresó que éste era su primer embarazo. Sobre el particular se coincide con Mendoza et al. (2016) al indicar que el embarazo no deseado es de por sí un riesgo para las adolescentes, en vista de que el 59\% de los casos terminan en nacimientos, pero, el $41 \%$ culminan en abortos y $14 \%$ de éstos son involuntarios.

Los datos de este estudio también se encuentran acordes con los hallazgos de Blanquet-García et al. (2016) debido a que el 78.1\% de las madres de ese estudio indicó que el embarazo no era deseado; y con los resultados del estudio realizado por Ayala y 
Toncel (2015) con madres adolescentes de Santa Marta, en donde el 90\% de las jóvenes manifestó que no deseaba quedar embarazada. Pero se percibe que recibieron apoyo afectivo de parte de su compañero durante el embarazo. Hallazgos que podrían reflejar mayor bienestar de las madres adolescentes que participaron en el estudio porque el soporte afectivo de la pareja es fundamental, le permite vivir la experiencia con menos miedos, mayor serenidad y más estabilidad. Se comparten opiniones con Castro y López (2014) quienes afirman que cuando la pareja de las madres adolescentes acepta el embarazo, se reducen los sentimientos de angustia y temor no solo hacia el parto sino también al futuro incierto.

Teniendo en cuenta los riesgos que implican la maternidad a temprana edad sobre la salud física y mental de la adolescente, así como para su propio bienestar, proyectos de vida y oportunidades futuras se concuerda con diversos estudios que plantean la necesidad de reducir el embarazo no deseado en las madres adolescentes de América Latina y el Caribe (CEPAL, 2018; OMS, 2018; UNFPA, 2018; UNFPA, 2019).

En líneas generales se concuerda con Posada (2014) quien afirma que el embarazo adolescente no es una opción sino una falta de opciones. Esto merece que se reflexione en torno al tema de la cultura en Santa Marta, debido a que el embarazo es una opción para salir de casa. Entre las novedades del proyecto se muestran hallazgos de las adolescentes encuestadas que estaban en el rango de edad entre los 12 y 14 años, atendiendo a lo mencionado por la OMS, la OPS, la UNFPA y la UNICEF en 2016 sobre los escasos datos sobre embarazos en menores de 15 años, donde muchos no son reportados. Los datos de este estudio permitirán al Sistema Nacional de Bienestar Familiar de la ciudad de Santa Marta conocer cuál debe ser el accionar de cada institución para abordar esta problemática. A partir de este estudio se solicitó a los investigadores realizar otro estudio en menores de 12 años para crear un programa de prevención desde la primera infancia. Se pretende impactar en la prevención de esta problemática con los siguientes productos elaborados: Cartilla de talleres educativos, una página web de consulta, y un video psicoeducativo sobre sexualidad responsable en la adolescencia.

\section{REFERENCIAS}

Blanquet-García, J., Montoya-Cázares, A., y Carranza-Lira-S. (2016). Características sociodemográficas de la adolescente embarazada en un hospital de alta especialidad. Revista Médica del Instituto Mexicano del Seguro Social, 54(3), 238-241.

Botero-Botero, L.D., y Castrillón-Osorio, L.C. (2015). La experiencia de la parternidad en adolescentes. Revista Virtual Universidad Católica del Norte, 46, 89-101.

Blos, P. (2011). Los comienzos de la adolescencia. Buenos Aires, Argentina: Amorrortu editores.

Carvajal, R., Valencia, H.L., y Rodríguez, RM. (2017). Factores asociados al embarazo en adolescentes de 13 a 19 años en el municipio de Buenaventura, Colombia. Revista 
Universitaria Industrial Santander Salud, 49(2), 290-300. http://dx.doi.org/10.18273/revsal.v49n2-2017002

Castro, M.D., y López, M.E. (2014). Embarazo en adolescentes. Diagnóstico sobre el acceso de adolescentes embarazadas a los servicios públicos de salud sexual y reproductiva en la ciudad de El Alto. La Paz: CIDES UMSA-UNFPA.

Comisión Económica para América Latina y el Caribe (2018). América Latina y el Caribe a 30 años de la Aprobación de la Convención sobre los Derechos del Niño. Santiago Chile. Naciones Unidas. Recuperado de: https://repositorio.cepal.org/bitstream/ handle/11362/44271/1/S1800977_es.pdf

Fondo de Población de las Naciones Unidas (2018). Una misión Común: menos embarazos en la Adolescencia en América Latina y el Caribe. Recuperado de: https://lac.unfpa.org/es/news/una-misi\%C3\%B3n-com\%C3\%BAn-menos-embarazosen-la-adolescencia-en-am\%C3\%A9rica-latina-y-el-caribe.

Fondo de Población de las Naciones Unidas (2019). Más de 19 mil embarazos en niñas y adolescentes, durante el año 2017 en El Salvador. Recuperado de: https://lac.unfpa.org/es/news/m\%C3\%A1s-de-19-mil-embarazos-en-ni\%C3\%B1as-yadolescentes-durante-el-a\%C3\%B1o-2017-en-el-salvador.

García, A., y González, M. (2018). Factores de riesgo asociados a embarazadas adolescentes. Revista de Ciencias Médicas de Pinar del Rio, 22(3), 416-427.

Gómez-Mercado, C., y Mejía-Sandoval, G. (2017). Prevalencia de embarazos y características demográficas, sociales, familiares, económicas de las adolescentes, Carepa, Colombia. CES Salud Pública, 25-33.

Huanco, D., Ticona, M., Ticona, M., y Huanco, F. (2012). Frecuencia y repercusiones maternas y perinatales del embarazo en adolescentes atendidas en hospitales del Ministerio de Salud del Perú, año 2008. Revista Chilena de Obstetricia y Ginecología, 77(2), 122-128.

Ley 1098 de noviembre del 2006. Código de infancia y adolescencia. Recuperado de: https://www.oas.org/dil/esp/Codigo_de_la_Infancia_y_la_Adolescencia_Colombia.pdf

López, F.A.M. (2014). Adolescencia: Límites imprecisos. Madrid: Alianza.

Mazuera-Arias, R., Trejos-Herrea, A.M., y Reyes-Ruiz, L. (2017). Percepción del embarazo adolescente en el Departamaneto Norte de Santander, Colombia. Revista de Salud Pública, 19(6), 733-738.

Mendoza, L.A., Claros, D.I., y Peñaranda, C.B. (2016). Actividad sexual temprana y embarazo en la adolescencia: estado del arte. Revista Chilena de Obstetricia y Ginecología, 81(3), 243-253.

Mesa para la concertación de la lucha contra la pobreza (2018). El embarazo en adolescentes y niñas en el Perú. Recuperado de http://mesagen.pe/download/de_aliados/MCLCP _Alerta_embarazo_adolescente.pdf

Ministerio de Salud Protección Social, Asociación Probienestar de la Familia Colombiana. (2015). Encuesta Nacional de Demografía y Salud. Bogotá, 2017. Recuperado de: http://microdata.worldbank.org/index.php/catalog/2834/related_materials.

Observatorio de la juventud en Iberoamérica (2018). El embarazo en adolescentes: INDH 2017. Recuperado de: https://www.observatoriodelajuventud.org/el-embarazo-en-adolescentesinforme-de-desarrollo-humano-en-republica-dominicana/ 
Organización Mundial de la Salud (2018). El embarazo en la adolescencia. Ginebra: Organización Mundial de la Salud.

Organización Mundial de la Salud, Organización Panamericana de la Salud, Fondo de Población de las Naciones Unidas y Fondo de las Naciones Unidas para la Infancia (2018). Acelerar el progreso hacia la reducción del embarazo en la adolescencia en América Latina y el Caribe. Informe de consulta técnica. Washington, D.C., EE. UU.

Organización Panamericana de Salud (2018). La salud de los adolescentes y jóvenes en la región de las américas. La aplicación de la estrategia y el plan de acción regionales sobre la salud de los adolescentes y jóvenes (2010-2018). Informe Resumido. Washington DC. Recuperado de: https://www.paho.org/informe-salud-adolescente-2018/images/ files/PAHO_Summary_Spa.pdf.

Pacheco-Sánchez, C. (2015). Embarazo en menores de quince años: Los motivos y la redefinición del curso de vida. Salud Púclica de México, 58(1), 56-61.

Pinzón.Rondon, A., Ruiz.Sternberg, A., Aguilera-Otalvaro, P., y Abril-Basto, P. (2018). Factores asociados al inicio de vida sexual y al embarazo adolescente en Colombia. Estudio de corte tranversal. Revista Child Ostetric Ginocological, 83(5), 487-489.

Posada, C. (2014). Embarazo en la adolescencia: no una opción, sino una falta de opciones. Revista Sexología y Sociedad, 10(25), 4-10.

Rojas, M., Méndez, R., y Montero, L. (2016) Salud sexual y reproductiva en adolescentes: la fragilidad de la autonomía. Hacia Promoción y Salud, 21(1), 52-62. doi:10.17151/hpsal.2016.21.1.6

Sanabria, J., y Fernández, Z. (2019). Factores de riesgo del embarazo en adolescentes y jóvenes, Bata, Guinea Ecuatorial. Revista de Ciencias Médicas, 23(1), 119-134.

Tafur-Bonilla, Y.A., Barbosa-Campos, O.L. y Méndez-Ortiz, E.L. (2018). Determinantes del embarazo en adolescentes y su impacto en los ejes fundamentales de ingreso y nivel educativo. Cooperativismo y Desarrollo, 113(26), 1-22. doi:10.16925/co.v26i113.2191

Urribarri, R. (2016). Adolescencia y clínica psicoanalítica. Buenos Aires, Argentina: Fondo de Cultura Económica.

Recibido: 5 de octubre de 2019 Recepción Modificaciones: 22 de noviembre de 2019 Aceptado: 23 noviembre de 2019 